\title{
MULTI-VIEW THREE-DIMENSIONAL IMAGE MONTAGING \& SIGNAL ATTENUATION CORRECTION FOR MAXIMIZING THE IMAGING DEPTH AND LATERAL EXTENT OF CONFOCAL MICROSCOPES
}

\author{
Omar Al-Kofahi ${ }^{1}$, Ali Can ${ }^{1}$, Sharie Lasek ${ }^{2}$, Donald H. Szarowski ${ }^{2}$, James N. Turner ${ }^{1,2}$, Badrinath Roysam ${ }^{1}$ \\ ${ }^{1}$ Rensselaer Polytechnic Institute, Troy, New York 12180-3590, USA. \\ ${ }^{2}$ The Wadsworth Center, NY State Department of Health, Albany, NY 12201-0509, USA.
}

Confocal Microscopy is valuable for its ability to image thick sections of intact tissue. A compelling need is to image biological structures (e.g. neurons) that are much larger than the conventional three-dimensional (3-D) field of view. We describe a combination of specimen preparation, imaging, and image processing methods that extend not only the lateral dimensions, but also the axial depth. We're also addressing the compelling need of improving the quantitative accuracy, and minimizing the possibility of missing important structures/phenomena due to non-uniform attenuation of the optical signal within the specimen. Our method is designed to work with existing instrumentation.

The axial extent of the image is increased by mounting the specimen in a symmetric manner between a pair of coverslips, and collecting two images. The first is collected in the routine way and the second after flipping the sample upside-down. These two views are separated roughly, but not exactly, by $180^{\circ}$ in the axial direction. The image structures in these views are registered precisely using fully automated and robust algorithms. Then, signal-processing algorithms based on modeling of the optical propagation of the epi-illumination and fluorescence signals through the specimen are used to compensate for the depth-dependent signal attenuation in the 3-D volume[2]. Previous methods for depth attenuation correction work by amplifying the signal from deeper parts of the specimen using an estimate of the signal extinction factor, and may need additional instrument setup [11]. In these methods, amplification of noise is an unavoidable artifact. Our approach has the advantage of exploiting data from multiple views to provably improve the signal to noise ratio and correct for photobleaching, while also accounting for the optical non-uniformity of the specimen. The noise variance of the montaged image is less than that for either individual view, corrected conventionally, and no additional instrument setup is needed.

Accurate image registration algorithms are critical to the success of the two-view approach outlined above [1]. First, there is an unavoidable and unknown tilt error of roughly $5^{\circ}$. The direction is unknown and uncontrollable. So, the registration algorithm must accommodate a 3-D rotation, along with the usual 3-D translation. Second, confocal images are non-isotropic, the axial resolution being lower than the lateral. Thus, non-isotropic scaling should be accounted for. Third, to maximize the lateral extent, the registration algorithm must be able to perform well with a small degree of overlap. Fourth, spatial aberrations must be accounted for. Finally, the algorithm must be stable and broadly applicable to a variety of specimens.

The above requirements were met with an innovative 3-D affine [7] registration algorithm. When the aberrations are small, assuming a well-adjusted instrument, this method was found to deliver a combination of stability, transformation flexibility and handling of aberrations. Our algorithm improves upon prior methods in several ways [3,4]. First, it achieves high accuracy (average error $<1.5$ voxels, equivalent to $0.55 \mu \mathrm{m}$ ) by using landmarks [6], instead of intensity correlations (Fig. 1). Second, it uses a hierarchy of models, starting with a rigid initial estimate with only 6 degrees of freedom (3-D rotation and 3-D translation) and then estimates a 3-D affine transformation with 12 degrees of freedom accounting for 3-D rotation, 3-D translation, non-isotropic scaling, and modest mechanical inconsistencies [2, 7, 8]. Third, it incorporates robust statistical methods to achieve accurate registration in the face of inaccurate and missing landmarks, and to eliminate instabilities due to the high dimensionality of affine models [9, 10]. Fourth, it is fully automated, even estimating the initial registration from the extracted landmarks. Fifth, it performs well with small overlapping volumes; in fact, minimum of only one common dendritic branching point landmark is sufficient. Finally, it is computationally efficient, taking less than a minute on a $900 \mathrm{MHz}$ Pentium III computer for registering two roughly $70 \mathrm{MB}$ images. The registration errors are a combination of modeling, estimation, discretization and tracing errors. Finally, our algorithm is broadly applicable to images of structures with distinctive point, line, and surface landmarks, implying broad applicability of the overall technique.

A sample result is shown in Fig. 2, 3. The figures show dye injected (4\% Alexa 594, Molecular Probes) neurons from a Wistar rat brain. The $250 \mu \mathrm{m}$ sections were mounted in $95 \%$ buffered glycerol with $1 \% \mathrm{n}$-propylgallate to minimize distortion. Images were collected using a NORAN Oz confocal mounted on an Olympus IX-70 inverted infinity corrected microscope, and a long-working-distance water immersion $\times 40$ lens (NA 1.15) with a field size of $192 \times 180 \mu \mathrm{m}$ 
and $0.375 \mu \mathrm{m} /$ pixel. Fig. 2 illustrates depth attenuation correction along the axial direction. The two views are aligned for better viewing. Structures that are highly attenuated in the first view (far from objective) are less attenuated in the second view (close to objective) and vice versa. The reconstructed image contains all the structures in the two views corrected for attenuation and photobleaching. Fig. 3 shows a 3-D rendering result for a neuron that is larger than the field of view of the microscope. The specimen was prepared symmetrically, the first image was recorded and then the specimen was translated on the $x-y$ stage to capture the rest of the neuron and the second image was recorded. Afterwards, the specimen was flipped as described above and two more images were recorded as with before flipping. The four images were montaged together, and the depth attenuation and photobleaching was corrected using the two-view correction. The reconstructed images are more faithful qualitative visualization of the specimen's structure and are quantitatively more accurate providing a rigorous basis for automated image analysis.

\section{References}

[1] Becker D. E., et al., (1996), Cytometry, vol. 25, no. 3, pp. 235-245.

[2] Can, A. et al., (1999), Proc. IEEE Comp. Society Conf on Comp. Vision \& Pattern Recognition., pp. $286-292$.

[3] Pavel V. B., Annica D. (1995), Journal of NeuroImage, vol. 2, no. 3, pp. 201-207

[4] Capek, M., and Krekule, I., (1999), IEEE Trans. on information tech. in biomedicine, vol. 3, no. 2, pp. $119-124$.

[5] Can, A., et al., (2000), Proceedings Microscopy and Microanalysis, vol. 6, pp. 818-819.

[6] Al-Kofahi K. et al, IEEE Transactions on Information Technology in Biomedicine (accepted, in press), 2002.

[7] Hartley R. and Zisserman A. (2000), Multiple View Geometry in Computer Vision, Cambridge University Press

[8] Kanatani, K. (1994), IEEE Trans. on PAMI, vol. 16 no. 5, pp. 543-549.

[9] Stewart, C.V. (1999), SIAM Rev., vol. 41 no. 3, pp. 513-537.

[10] Besl, P.J. \& McKay, N.D. (1992), IEEE Trans. on PAMI, vol. 14, no. 2, pp. 239-256.

[11] Mainen Z. F., et al.,(1999), Methods: A Companion to Methods in Enzymology, 18, no. 2, $231-239$.

[12] This work supported by the NSF Center for Subsurface Sensing \& Imaging Systems (EEC-9986821).

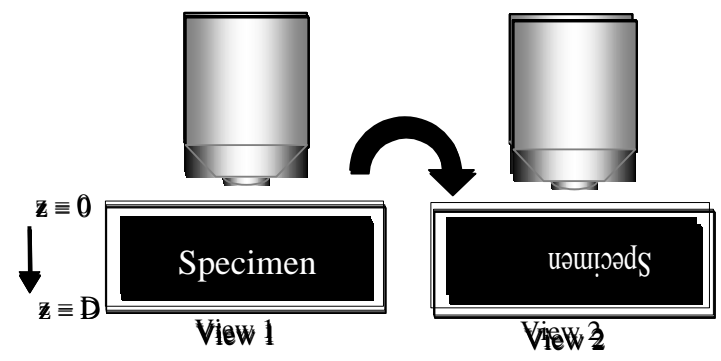

FIG 1: Illustrating the two-view imaging method. The specimen is mounted between cover slips to enable symmetric two-sided imaging.

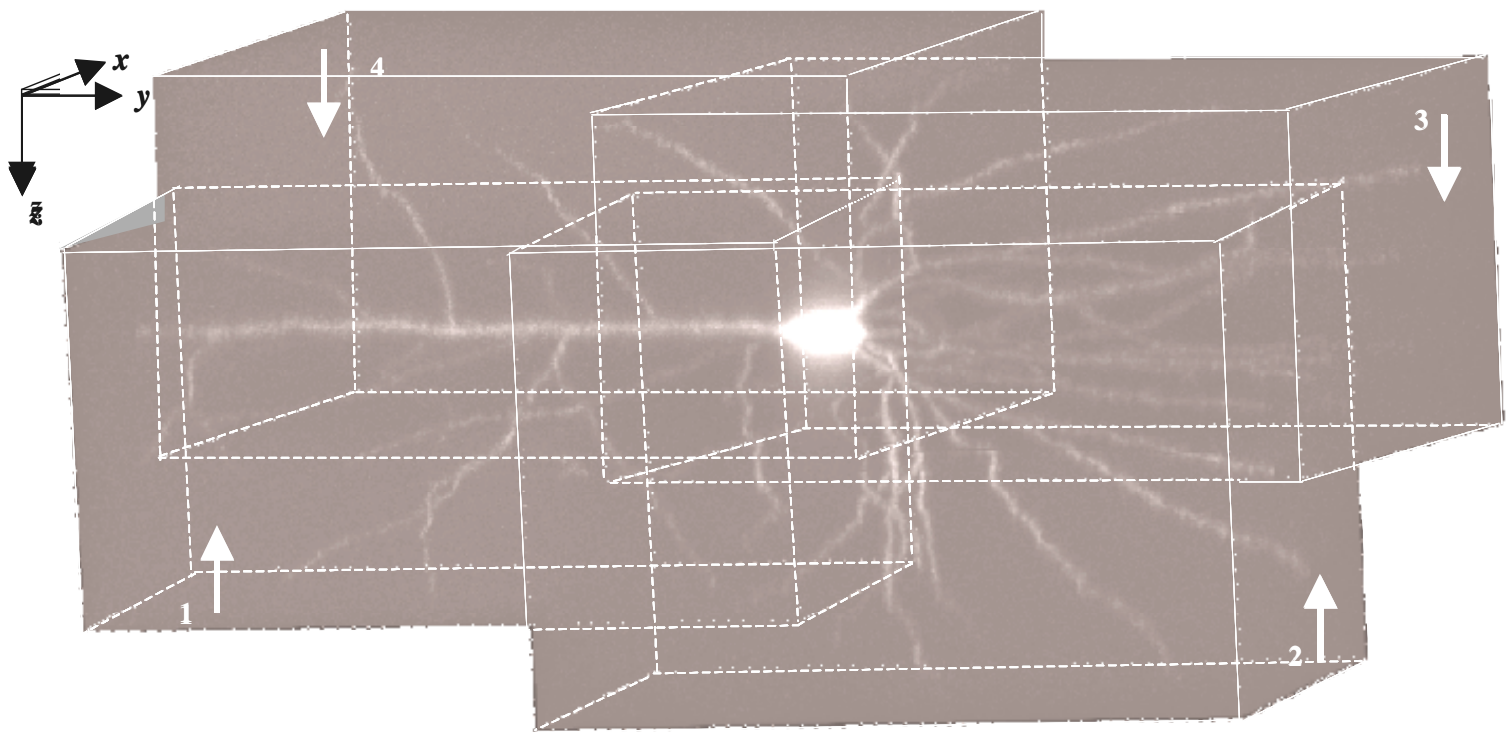

FIG 3 (left): A 3D volume rendering of a twosided attenuationcorrected montage of a large dyeinjected neuron reconstructed from four views, two from each direction. The individual (192x180x130 $\mu \mathrm{m})$ image stacks are highlighted. The arrows indicate the imaging direction. 